International Mathematical Forum, Vol. 8, 2013, no. 16, 789 - 799 HIKARI Ltd, www.m-hikari.com

\title{
Classes of Universal Graphs Definable by Universal Graph Algebra Quasi-Identities
}

\author{
P. Jampachon and T. Poomsa-ard \\ Department of Mathematics, Faculty of Science \\ Khon Kaen University, Khon Kaen 40000, Thailand \\ tiang@kku.ac.th
}

Copyright (c) 2013 P. Jampachon and T. Poomsa-ard. This is an open access article distributed under the Creative Commons Attribution License, which permits unrestricted use, distribution, and reproduction in any medium, provided the original work is properly cited.

\begin{abstract}
Universal graph algebras establish a connection between universal graphs (i.e. $n_{i}$-ary relations) and universal algebras. A universal graph theoretic characterization of universal graph quasi-varieties, i.e. classes of universal graphs definable by universal graph quasi-identities, is given. The results give rise to a structure theorem of Birkhoff-type: A class of finite undirected universal graphs is a universal graph quasivarieties if and only if it is closed with respect to isomorphisms, induced subgraphs, finite disjoint unions and homogeneous subproducts. Some examples are also considered.
\end{abstract}

Mathematics Subject Classification: 05C25, 08B15

Keywords: universal graphs, universal graph algebras, universal graph varieties, universal graph quasi-varieties, terms, homogeneous subproducts, directed products

\section{Introduction}

Graph algebras have been invented in [5] to obtain examples of nonfinitely based finite algebras. To recall this concept, let $G=(V, E)$ be a (directed) graph with the vertex set $V$ and the edge set $E \subseteq V \times V$. Define the graph 
algebra $A(G)$ corresponding to $G$ to have the underlying set $V \cup\{\infty\}$, where $\infty$ is a symbol outside $V$, and two basic operations, a nullary operation pointing to $\infty$ and binary one denoted by juxtaposition, given for $u, v \in V \cup\{\infty\}$ by

$$
u v=\left\{\begin{array}{cl}
u, & \text { if }(u, v) \in E, \\
\infty, & \text { otherwise }
\end{array}\right.
$$

Universal graph algebras have been defined in [3] to obtain some basic properties of universal graph algebras. To recall this concept, let $G=(V, E)$ be a multi graph with the vertex set $V$ and the edge set $E$, an edge in $E$ is an ordered pair of vertices (not necessarily distinct) of $V$ and let $\tau=\left(n_{i}\right)_{i \in I}, n_{i} \geq$ 2 be a sequence of natural number. A function $f_{i}: V^{n_{i}} \rightarrow V$ is called an $n_{i}$-ary operation defined on $V$, and is said to have arity $n_{i}$. For convenient we label all edges of $E$ to be distinct. Let $E_{f_{i}} \subseteq V^{n_{i}}$, where $\left(v_{1}, v_{2}, \ldots, v_{n_{i}}\right) \in E_{f_{i}}$ if and only if $\left(v_{1}, v_{2}\right),\left(v_{2}, v_{3}\right), \ldots,\left(v_{n_{i}-1}, v_{n_{i}}\right) \in E$ and the labels of them are all different, further if $e_{f_{i}}=\left(v_{1}, v_{2}, \ldots, v_{n_{i}}\right) \in E_{f_{i}}$ and $e_{f_{j}}=\left(u_{1}, u_{2}, \ldots, u_{n_{j}}\right) \in E_{f_{j}}$, then the intersection of the label set of $\left(v_{1}, v_{2}\right),\left(v_{2}, v_{3}\right), \ldots,\left(v_{n_{i}-1}, v_{n_{i}}\right)$ and the label set of $\left(u_{1}, u_{2}\right),\left(u_{2}, u_{3}\right), \ldots,\left(u_{n_{j}-1}, u_{n_{j}}\right)$ is an empty set. In this case $G=\left(V,\left(E_{f_{i}}\right)_{i \in I}\right)$ is called an universal graph of type $\tau=\left(n_{i}\right)_{i \in I}, n_{i} \geq 2$. Define the universal graph algebra $A(G)$ of type $\tau=\left(0,\left(n_{i}\right)_{i \in I}\right), n_{i} \geq 2$ corresponding to the universal graph $\bar{G}$ with the underlying set $V \cup\{\infty\}$, where $\infty$ is a symbol outside $V$, and basic operations, nullary operation pointing to $\infty$ and $n_{i}$-ary operations $f_{i}, i \in I$, given for elements of $(V \cup\{\infty\})^{n_{i}}, n_{i} \geq 2$ by

$$
f_{i}\left(v_{1}, v_{2}, \ldots, v_{n_{i}}\right)= \begin{cases}v_{1}, & \text { if }\left(v_{1}, v_{2}, \ldots, v_{n_{i}}\right) \in E_{f_{i}}, \\ \infty, & \text { otherwise. }\end{cases}
$$

In [4] graph quasi-varieties had been investigated for finite undirected graphs in order to get graph theoretic results (structure theorems) from universal algebra via graph algebras. In the present paper these investigations are extended to finite undirected universal graphs. We ask for an universal graph theoretic characterization of universal graph quasi-varieties, i.e. the classes of universal graphs with can be defined by quasi-identities for their corresponding universal graph algebras. The answer is a theorem of Birkhoff-type, which uses graph theoretic closure operations. A class of finite undirected universal graphs is quasi-equational (i.e., an universal graph quasi-variety) if and only if it is closed with respect to isomorphisms, induced subgraphs, finite disjoint unions and homogeneous subproducts.

\section{Preliminaries}

We recall the following terminology and notations. Let $G=\left(V,\left(E_{f_{i}}\right)_{i \in I}\right)$ be an universal graph of type $\tau=\left(n_{i}\right)_{i \in I}, n_{i} \geq 2$. G is called undirected, if for 
each $E_{f_{i}}, i \in I$ is a symmetric relation. An edge $e_{f_{i}}=\left(v_{1}, v_{2}, \ldots, v_{n_{i}}\right) \in E_{f_{i}}$ is called a loop if $v_{1}=v_{n_{i}}$. Let $V^{\prime}(G)$ be the set of all vertices $v \in V(G)$ which is an initial point or a terminal point of an edge in $E(G), E^{\prime}(G)$ be the set of all order pairs $(u, v) \in V(G) \times V(G)$ where $u, v$ are initial and terminal points of an edge in $E(G)$, respectively. A universal graph $G^{*}$ is called a subgraph of $G$ if $V\left(G^{*}\right) \subseteq V(G)$ and $E_{f_{i}}\left(G^{*}\right) \subseteq E_{f_{i}}(G), i \in I$. A universal graph $G^{*}$ is called an induced subgraph of $G$ if $V\left(G^{*}\right) \subseteq V(G)$ and $E_{f_{i}}\left(G^{*}\right)=E_{f_{i}}(G) \cap\left(V\left(G^{*}\right)\right)^{n_{i}}, i \in I$. Given universal graph $G_{i}, i \in I$, the direct product $G=\prod_{i \in I} G_{i}$ is the universal graph $G$ with $V(G)=\prod_{i \in I} V\left(G_{i}\right)$ and $E_{f_{i}}(G)=\left\{\left(a_{1}, a_{2}, \ldots, a_{n_{i}}\right) \in(V(G))^{n_{i}} \mid\left(a_{1}(j), a_{2}(j), \ldots, a_{n_{i}}(j)\right) \in E_{f_{i}}\left(G_{j}\right), j \in\right.$ $I\}, i \in I$. Here, for $a \in V(G)$, let $a(j)$ denote the $j$-component: $a=(a(j))_{j \in I}$. Assume the sets $V\left(G_{i}\right), i \in I$ to be pairwise disjoint (otherwise make the sets $V\left(G_{i}\right)$ disjoint i.e. use $\left.V\left(G_{i}\right) \times\{i\}\right)$, then the disjoint union $G=\cup_{i \in I} G_{i}$ is simply the union of the universal graphs $G_{i}$, i.e. $V(G)=\cup_{i \in I} V\left(G_{i}\right), E_{f_{j}}(G)=$ $\cup_{i \in I} E_{f_{j}}\left(G_{i}\right), j \in I$. Products or unions are called finite if $I$ is finite. A mapping $h: V(G) \rightarrow V\left(G^{*}\right)$ is a homomorphism from a universal graph $G$ into a graph $G^{*}$ if for all $a, b \in V^{\prime}(G),\left(a_{1}, a_{2}, \ldots, a_{n_{i}}\right) \in E_{f_{i}}(G), i \in I, a=a_{1}, b=a_{n_{i}}$ implies $\left(h\left(a_{1}\right), h\left(a_{2}\right), \ldots, h\left(a_{n_{i}}\right)\right) \in E_{f_{i}}\left(G^{*}\right)$. The homomorphism $h$ is called strong if $\left(a_{1}, a_{2}, \ldots, a_{n_{i}}\right) \notin E_{f_{i}}(G)$ implies $\left(h\left(a_{1}\right), h\left(a_{2}\right), \ldots, h\left(a_{n_{i}}\right)\right) \notin E_{f_{i}}\left(G^{*}\right)$. An isomorphism (universal graph isomorphism) is a bijective strong homomorphism. $H\left(G, G^{*}\right)$ denotes the set of all homomorphism from $G$ into $G^{*}$. For class $\mathcal{R}$ of universal graphs, let $I \mathcal{R}, S \mathcal{R}, P \mathcal{R}, P_{f} \mathcal{R}, U \mathcal{R}, U_{f} \mathcal{R}$ denote the classes of all isomorphic copies, induced subgraphs, direct products, finite direct products, disjoint unions, and finite disjoint unions of members of $\mathcal{R}$, respectively. Let $\mathcal{G}_{d}, \mathcal{G}_{d f}$ and $\mathcal{G}_{u f}$ be the class of directed universal graphs, the class of finite directed universal graphs and the class of finite undirected universal graphs, respectively. We omit the index $f$ (= finite), if also infinite universal graphs are to be considered. For all universal graphs $G$ under consideration, let $G=\left(V,\left(E_{f_{i}}\right)_{i \in I}\right)$ as defined in section 1 (introduction). The algebra $A(G)=\left(V(G) \cup\{\infty\},\left(f_{i}\right)_{i \in I}, \infty\right)$ is called the universal graph algebra of $G$. For universal graphs $G_{1}$ and $G_{2}, h: G_{1} \rightarrow G_{2}$ is a strong homomorphism if and only if $h: A\left(G_{1}\right) \rightarrow A\left(G_{2}\right)$ (extended by $h(\infty)=\infty$ ) is a homomorphism of the corresponding universal graph algebras.

Let $W_{\tau}(X), \tau=\left(0,\left(n_{i}\right)_{i \in I}\right), n_{i} \geq 2$, be the set of all terms over the alphabet $X=\left\{x_{1}, x_{2}, x_{3}, \ldots\right\}$ defined inductively as follows:

(i) every variable $x_{i}, i=1,2,3, \ldots$, and $\infty$ are terms,

(ii) if $t_{1}, t_{2}, \ldots, t_{n_{i}}$ are terms, then $f_{i}\left(t_{1}, t_{2}, \ldots, t_{n_{i}}\right)$ is a term, where $f_{i}$ is an $n_{i}$-ary operation.

$W_{\tau}(X)$ is the set of all terms which can be obtained from (i) and (ii) in finitely many steps. The leftmost variable of a term $t$ is denoted by $L(t)$ and the rightmost variable of a term $t$ is denoted by $R(t)$. The terms in which the symbol $\infty$ 
occurs are called a trivial terms. These terms evaluate to $\infty$ in every universal graph algebras. To every non-trivial term $t$ of type $\tau=\left(0,\left(n_{i}\right)_{i \in I}\right), n_{i} \geq 2$ we assign an universal graph $G(t)=\left(V(t),\left(E_{f_{i}}(t)\right)_{i \in I}\right)$, where the vertex set $V(t)$ is the set of all variables occurring in $t$ and each $E_{f_{i}}(t), i \in I$ is defined inductively by

$$
\begin{gathered}
E_{f_{i}}(t)=\phi, i \in I \text { if } t \text { is a variable and } E_{f_{i}}\left(f_{i}\left(t_{1}, t_{2}, \ldots, t_{n_{i}}\right)\right)= \\
E_{f_{i}}\left(t_{1}\right) \cup E_{f_{i}}\left(t_{2}\right) \cup \ldots \cup E_{f_{i}}\left(t_{n_{i}}\right) \cup\left\{\left(L\left(t_{1}\right), L\left(t_{2}\right), \ldots, L\left(t_{n_{i}}\right)\right)\right\},
\end{gathered}
$$

when $t=f_{i}\left(t_{1}, t_{2}, \ldots, t_{n_{i}}\right)$ is a compound term and $L\left(t_{1}\right), L\left(t_{2}\right), \ldots, L\left(t_{n_{i}}\right)$ are the leftmost variables of $t_{1}, t_{2}, \ldots, t_{n_{i}}$ respectively. Formally, we assign the empty graph $\phi$, to every trivial term $t$.

For convenience we will introduce some more notations about universal graph in the following way: Let $G=\left(V,\left(E_{f_{i}}\right)_{i \in I}\right)$ be an universal graph. For any $u, v \in V(G)$, we say that there exists a dipath from $u$ to $v$ in $G$ if there exists a sequence of order pairs $\left(a_{1}, b_{1}\right),\left(a_{2}, b_{2}\right), \ldots,\left(a_{n}, b_{n}\right)$ in $E^{\prime}(G)$ such that $u=a_{1}, b_{1}=a_{2}, \ldots, b_{n-1}=a_{n}$ and $b_{n}=v$. For any $u \in V(G)$, the set of $u$ and all $v \in V(G)$ such that there exists a dipath from $u$ to $v$ in $G$, is denoted by $A_{G}(u)$. If $A_{G}(u)=V(G)$, then we say that $G$ is a rooted universal graph with root $u$. We see that $L(t)$ is a root of the universal graph $G(t)$ and the pair $(G(t), L(t))$ is the rooted universal graph corresponding to $t$.

Given $t \in W_{\tau}(X), G \in \mathcal{G}_{d}$ and a mapping (assignment) $h: V(t) \rightarrow V(G) \cup$ $\{\infty\}$, let $h(t)$ denote the value of $t$ in $A(G)$ when every variable $x \in V(t)$ is substituted by $h(x)$. Let $s, t \in W_{\tau}(X \overline{)}$. A universal graph $G$ satisfies the equation $s \approx t$ ( or $s \approx t$ holds in $G$ ), denoted by $G=s \approx t$, if $h(s)=h(t)$ holds in $A(G)(G \models h(s) \approx h(t))$ for every assignment $h: V(s) \cup V(t) \rightarrow V(G) \cup\{\infty\}$. For a set $\Sigma$ of equations and an assignment $h: V(\Sigma) \rightarrow V \cup\{\infty\})(V(\Sigma)$ denotes the set of variables occurring in $\Sigma$ ) we write $G \models h(\Sigma)$ if for all $s \approx t \in \Sigma, G \models h(s) \approx h(t)$ and we write $G \models \Sigma$ if $G \models s \approx t$ for all $s \approx t \in \Sigma$.

Let $t \in W_{\tau}$ be a term. We write $t\left(x_{i_{1}}, x_{i_{2}}, \ldots, x_{i_{n}}\right)$ if $x_{i_{1}}, x_{i_{2}}, \ldots, x_{i_{n}}$ are the variables which occur in $t$. Given a term $t\left(x_{0}, x_{1}, \ldots, x_{n}\right)$, an universal graph $G$ and a mapping $h: V(t) \rightarrow V(G) \cup\{\infty\}$, we define $h(t)=$ $t\left(h\left(x_{0}\right), h\left(x_{1}\right), \ldots, h\left(x_{n}\right)\right)$ (the right hand side has to be computed in $\left.A(G)\right)$. Sometimes we call $h$ an evaluation of the variables.

In [3], proved the following propositions:

Proposition 2.1. Let $G=\left(V,\left(E_{f_{i}}\right)_{i \in I}\right)$ be an universal graph of type $\tau=$ $\left(n_{i}\right)_{i \in I}, n_{i} \geq 2$ and let $h: X \longrightarrow V \cup\{\infty\}$ be an evaluation of the variables. Consider the canonical extension of $h$ to the set of all terms. Then there holds: if $t$ is a trivial term, or if $h$ takes the value $\infty$ on $X$, then $h(t)=\infty$. Otherwise, if $h: G(t) \longrightarrow G$ is a homomorphism of graphs, then $h(t)=h(L(t))$, and if $h$ is not a homomorphism of graphs, then $h(t)=\infty$. 
Proposition 2.2. Let $s$ and $t$ be non-trivial terms from $W_{\tau}(X)$ with variables $V(s)=V(t)=\left\{x_{0}, x_{1}, \ldots, x_{n}\right\}$ and $L(s)=L(t)$. Then an universal graph $G=\left(V,\left(E_{f_{i}}\right)_{i \in I}\right)$ satisfies $s \approx t$ if and only if the universal graph algebra $A(G)$ has the following property: A mapping $h: V(s) \longrightarrow V \cup\{\infty\}$ is a homomorphism from $G(s)$ into $G$ iff it is a homomorphism from $G(t)$ into $G$.

Proposition 2.3. Let $s, t \in W_{\tau}(X), \tau=\left(0,\left(n_{i}\right)_{i \in I}\right), n_{i} \geq 2$ be terms. Then the non-trivial equation $s \approx t$ is an identity in the class of all universal graph algebras iff either both terms $s$ and $t$ are trivial or none of them is trivial, $G(s)=G(t)$ and $L(s)=L(t)$.

A quasi-identity $q$ is a finite set $\Sigma=\left\{s_{1} \approx t_{1}, \ldots, s_{n} \approx t_{n}\right\}$ of equations together with an equation $s \approx t$ : we use the notation $\Sigma \rightarrow s \approx t$ or $s_{1} \approx$ $t_{1} \wedge \ldots \wedge s_{n} \approx t_{n} \rightarrow s \approx t$. A graph $G$ satisfies the quasi-identities $q$, denoted by $G \models q$, if for every assignment $h: V(q) \rightarrow V(G) \cup\{\infty\}(V(q)$ denotes the set of variables occurring in $q$ ) the following implication holds:

$$
h\left(s_{1}\right) \approx h\left(t_{1}\right) \wedge \ldots \wedge h\left(s_{n}\right) \approx h\left(t_{n}\right) \rightarrow h(s) \approx h(t) .
$$

This will be denoted by $G \models h(\Sigma) \rightarrow h(s) \approx h(t)$ (or $G \models h(q)$ ). Note that every equation $s \approx t$ can be considered as a quasi-identity because $G \models s \approx$ $t \Leftrightarrow G=\infty \approx \infty \rightarrow s \approx t$. For $\mathcal{R} \subseteq \mathcal{G}_{d}$ and a set $\mathcal{Q}$ of quasi-identities (or equations) we write $\mathcal{R} \models \mathcal{Q}$ if $G \models q$ for all $q \in \mathcal{Q}$ and $G \in \mathcal{R}$.

\section{Characterization of universal graph quasi- varieties}

Definition 3.1. For a set $\mathcal{Q}$ of quasi-identities and a class $\mathcal{R}$ of universal $\operatorname{graphs}$, let $\operatorname{Mod}(\mathcal{Q})=\left\{G \in \mathcal{G}_{d} \mid G \models \mathcal{Q}\right\}$ and $\operatorname{Qid}(\mathcal{R})=\{q \mid q$ is a quasiidentity and $\mathcal{R} \models q\}$. We let $\operatorname{Qvar}(\mathcal{R})=\operatorname{Mod}(\operatorname{Qid}(\mathcal{R}))$ and for a given class $\mathcal{G}$ of universal graphs, $\operatorname{Qvar}_{\mathcal{G}}(\mathcal{R})=\mathcal{G} \cap \operatorname{Qvar}(\mathcal{R})$. A class of this form is called a quasi-equational or a universal graph quasi-variety in $\mathcal{G}$. In particular, $\operatorname{Qvar}_{\mathcal{G}}(\mathcal{R})$ is the universal graph quasi-variety generated by $\mathcal{R}$ in $\mathcal{G}$.

Remark 3.1. In general, universal graph quasi-varieties are not quasi-varieties in the usual universal-algebraic sense, e.g. the direct product of universal graph algebras is not a universal graph algebra. Clearly, $\operatorname{Qvar}_{\mathcal{G}}(\mathcal{R})$ consists of universal graphs from $\mathcal{G}$ whose universal graph algebras belong to the quasi-variety $I S P P_{u} \mathcal{R}^{*}$ (cf. [1] p. 219, Thm. 2.25, $\left.\mathcal{R}^{*}=\{A(G) \mid G \in \mathcal{R}\}\right)$. However, the most algebras in $I S P P_{u} \mathcal{R}^{*}$ are not universal graph algebras. Therefore it is reasonable to ask for an internal characterization of $\operatorname{Qvar}_{\mathcal{G}}(\mathcal{R})$ using operations on binary relations (= uiversal graphs) only to avoid ultraproducts and infinite universal graphs. 
Definition 3.2. A universal graph $G$ is called a homogeneous subproduct of a family $\left(G_{i}\right)_{i \in I}$ of universal graphs, if

(i) $G$ is an induced subgraph of $\prod_{i \in I} G_{i}$,

(ii) For all $a, b \in V^{\prime}(G)$ either $(a(i), b(i)) \in E^{\prime}\left(G_{i}\right)$ forall $i \in I$ or $(a(i), b(i)) \notin$ $E^{\prime}\left(G_{i}\right)$ forall $i \in I$.

For a set $\mathcal{R}$ of universal graphs, let $P_{h} \mathcal{R}\left(P_{h f} \mathcal{R}\right.$, resp.) denote the set of all homogeneous subproducts of families (finite families, resp.) of members of $\mathcal{R}$.

Proposition 3.1. Let $\mathcal{R}$ be the class of universal graphs and $G$ be a universal graph with $\left|V^{\prime}(G)\right| \geq 2$. Then $G \in I P_{h} \mathcal{R}$ if and only if for all $a, b \in V^{\prime}(G)$ with $a \neq b$ there exists an $R \in \mathcal{R}$ and a strong homomorphism $\phi: G \rightarrow R$ such that $\phi(a) \neq \phi(b)$. Moreover, if $G$ is finite, then $G \in I P_{h} \mathcal{R}$ implies $G \in I P_{h f} \mathcal{R}$. In case $\left|V^{\prime}(G)\right|=1, G \in I P_{h} \mathcal{R}$ if and only if there is a strong homomorphism $\phi: G \rightarrow R$.

Proof. $(\Rightarrow)$ If $G \subseteq \prod_{i \in I} R_{i}$ is a homogeneous subproduct, then every projection $p_{i}: G \rightarrow R_{i}: a \mapsto a(i)$ is a homomorphism which is strong by $(i i)$. Distinct $a, b \in V^{\prime}(G)$ must differ in at least one component $i \in I$, i.e. $p_{i}(a) \neq p_{i}(b)$.

$(\Leftarrow)$ Let $I=\left\{(a, b) \mid a \neq b, a, b \in V^{\prime}(G)\right\}$ and assume that there is a strong homomorphism $\phi_{(a, b)}: G \rightarrow R_{(a, b)} \in \mathcal{R}$ with $\phi_{(a, b)}(a) \neq \phi_{(a, b)}(b)$ for all $(a, b) \in I$. Let $B$ be the induced subgraph of $\prod_{i \in I} R_{i}$ with $V^{\prime}(B)=$ $\left\{\left(\phi_{i}(c)\right)_{i \in I} \mid c \in V^{\prime}(G)\right\}$. Then $B$ is a homogeneous subproduct. Since $\phi_{i}$ is a strong homomorphism, there exists $i \in I,\left(\phi_{i}(c), \phi_{i}\left(c^{\prime}\right)\right) \in E^{\prime}\left(R_{i}\right)$. So $\left(c, c^{\prime}\right) \in E^{\prime}(G)$, we get $\left(\phi_{i}(c), \phi_{i}\left(c^{\prime}\right)\right) \in E^{\prime}\left(R_{i}\right)$ for all $i \in I$. Moreover, $G$ is isomorphic to $B$ because $c \mapsto\left(\phi_{i}(c)\right)_{i \in I}$ is an isomorphism by construction, i.e. $G \in I P_{h} \mathcal{R}$. If $G$ is finite, this construction also gives a finite set $I$.

Lemma 3.1. Let $s$ and $t$ be non-trivial terms and let $B \subseteq \prod_{i \in I} G_{i}$ be a homogeneous subproduct of $\left(G_{i}\right)_{i \in I}$. Then for an assignment $h: V(s) \cup V(t) \rightarrow$ $V(B) \cup\{\infty\}$, we have $B \models h(s) \approx h(t)$ if and only if $G_{i} \models h_{i}(s) \approx h_{i}(t)$, where $h_{i}$ is the composition of $h$ with the $i$-th projection $p_{i}\left(h_{i}(x)=p_{i}(h(x))=\right.$ $(h(x))(i)$, and $h_{i}(x)=\infty$ if $\left.h(x)=\infty\right)$.

Proof. $(\Rightarrow)$ Suppose that $B \models h(s) \approx h(t)$. If $h(s)=\infty$ with $h(x)=\infty$, then $h(t)=\infty$. Suppose that $h(t)=\infty$ with $h(y)=\infty$. We have $h_{i}(x)=$ $\infty$ and $h_{i}(y)=\infty$. Hence $h_{i}(s)=\infty=h_{i}(t)$. Suppose that $h(t)=\infty$ and the restriction of $h$ on $V(t)$ is not a homomorphism. Hence there exists $(u, v) \in E^{\prime}(t)$ such that $(h(u), h(v)) \notin E^{\prime}(B)$. Since $B$ is a homogeneous subproduct, we have $(h(u)(i), h(v)(i)) \notin E^{\prime}\left(G_{i}\right)$. Therefore $h_{i}(t)=\infty$. We get $h_{i}(s)=\infty=h_{i}(t)$. Similarly, we can proof that if $h(s)=\infty$ and the restriction of $h$ on $V(s)$ is not a homomorphism, then $h_{i}(s)=\infty=h_{i}(t)$. Next, we suppose that $h(s) \neq \infty$. Then we have $h(t) \neq \infty$. Let $h(s)=h(x)$ 
and $h(t)=h(y)$. Hence $h(x)=h(y)$, i.e. $(h(x))(i)=(h(y))(i)$ for all $i \in I$. Therefore $h_{i}(s)=h_{i}(x)=p_{i}(h(x))=p_{i}(h(y))=h_{i}(y)=h_{i}(t)$.

$(\vDash)$ Suppose that $h_{i}(s)=h_{i}(t)$. If $h_{i}(s)=\infty$ with $h_{i}(x)=\infty$, then $h_{i}(t)=\infty$. Suppose that $h_{i}(t)=\infty$ with $h_{i}(y)=\infty$. Since $G(s)$ is connected, there is $z \in V(s)$ such that $(x, z) \in E^{\prime}(s)$ or $(z, x) \in E^{\prime}(s)$. Since $h(x)=\infty$, we have $(h(x), h(z)) \notin E^{\prime}(B)$ and $(h(z), h(x)) \notin E^{\prime}(B)$. Hence $h(s)=\infty$. Similarly, we have $h(t)=\infty$. Suppose that $h_{i}(t)=\infty$ and the restriction of $h_{i}$ on $V(t)$ is not a homomorphism. Then there exists $(u, v) \in E^{\prime}(t)$ such that $\left(h_{i}(u), h_{i}(v)\right) \notin E^{\prime}(B)$. Since $B$ is a homogeneous subproduct, we have $\left(h_{i}(u)(i), h_{i}(v)(i)\right) \notin E^{\prime}\left(G_{i}\right)$ and $(h(u), h(v)) \notin E^{\prime}(B)$. Therefore $h(t)=\infty$. We get $h(s)=\infty=h(t)$. In the same manner, we can proof that if $h_{i}(s)=\infty$ such that the restriction of $h_{i}$ on $V(s)$ is not a homomorphism, then $h(s)=$ $\infty=h(t)$. Suppose that $h_{i}(s) \neq \infty$. We have $h_{i}(t) \neq \infty$. Let $h_{i}(s)=h_{i}(x)$ and $h_{i}(t)=h_{i}(y)$. Hence $h_{i}(x)=h_{i}(y)$ i.e. $(h(x))(i)=(h(y))(i), i \in I$. Therefore $h(x)=h(y)$. We get $h(s)=h(x)=h(y)=h(t)$.

Lemma 3.2. Let $s$ and $t$ be non-trivial terms, $G$ be a disjoint union of $G_{1}$ and $G_{2}, h: V(s) \cup V(t) \rightarrow V(G) \cup\{\infty\}$ be an assignment and let $h_{i}: V(s) \cup V(t) \rightarrow$ $V(G) \cup\{\infty\}$ be defined by $h_{i}(x)=h(x)$ if $h(x) \in V\left(G_{i}\right)$ and $h_{i}(x)=\infty$ otherwise $(i \in\{1,2\})$. Then $h(s)=h(t)$ if and only if $h_{1}(s)=h_{1}(t)$ and $h_{2}(s)=h_{2}(t)$.

Proof. First we show that $h(s)=\infty$, if $h(V(s)) \nsubseteq V\left(G_{1}\right)$ and $h(V(s)) \nsubseteq$ $V\left(G_{2}\right)$. Suppose that $x, y \in V(s), h(x) \in V\left(G_{1}\right), h(x) \notin V\left(G_{2}\right), h(y) \in V\left(G_{2}\right)$ and $h(y) \notin V\left(G_{1}\right)$. Since $G(s)$ is a connected universal graph. Hence, there is an edge $e \in E(G(s))$ which $x$ and $y$ occur. But since $G_{1}$ and $G_{2}$ are disjoint, there is no edge $e^{\prime} \in h(V(s))$ which $h(x)$ and $h(y)$ occur. Therefore $h$ is not a homomorphism. We get $h(s)=\infty$. In the similar way we have $h(t)=\infty$ if $h(V(t)) \nsubseteq V\left(G_{1}\right)$ and $h(V(t)) \nsubseteq V\left(G_{2}\right)$. Moreover, from construction we see that $h_{i}(s)=h(s)$ if $h(V(s)) \subseteq V\left(G_{i}\right) \cup\{\infty\}, h_{i}(s)=\infty$ if $h(V(s)) \nsubseteq V\left(G_{i}\right)$ and $h_{i}(t)=h(t)$ if $h(V(t)) \subseteq V\left(G_{i}\right) \cup\{\infty\}, h_{i}(t)=\infty$ if $h(V(t)) \nsubseteq V\left(G_{i}\right)$. Now we will use these properties to show that $h(s)=h(t)$ if and only if $h_{1}(s)=h_{1}(t)$ and $h_{2}(s)=h_{2}(t)$. Clearly, if $h(s)=h(t)$, then $h_{1}(s)=h_{1}(t)$ and $h_{2}(s)=h_{2}(t)$. Suppose that $h_{1}(s)=h_{1}(t)$ and $h_{2}(s)=h_{2}(t)$ and let $h: V(s) \cup V(t) \rightarrow V(G) \cup\{\infty\}$ be an assignment. Since $h_{1}(s)=h_{1}(t)$, we have $h(V(s)) \subseteq V\left(G_{1}\right) \cup\{\infty\}$ if and only if $h(V(t)) \subseteq V\left(G_{1}\right) \cup\{\infty\}$ and since $h_{2}(s)=$ $h_{2}(t)$, we have $h(V(s)) \subseteq V\left(G_{2}\right) \cup\{\infty\}$ if and only if $h(V(t)) \subseteq V\left(G_{2}\right) \cup\{\infty\}$. If $h(V(s)) \subseteq V\left(G_{1}\right) \cup\{\infty\}$, then we have $h(V(t)) \subseteq V\left(G_{1}\right) \cup\{\infty\}$. Hence $h(s)=h_{1}(s)=h_{1}(t)=h(t)$. If $h(V(s)) \nsubseteq V\left(G_{1}\right)$, then $h(V(t)) \nsubseteq V\left(G_{1}\right)$ and if $h(V(t)) \nsubseteq V\left(G_{2}\right)$, then $h(V(s)) \nsubseteq V\left(G_{2}\right)$. Hence $h(V(s)) \nsubseteq V\left(G_{1}\right)$, $h(V(t)) \nsubseteq V\left(G_{2}\right)$ or $h(V(t)) \subseteq V\left(G_{2}\right)$. If $h(V(s)) \nsubseteq V\left(G_{1}\right)$ and $h(V(t)) \nsubseteq$ $V\left(G_{2}\right)$, then we get $h(V(s)) \nsubseteq V\left(G_{1}\right), h(V(s)) \nsubseteq V\left(G_{2}\right), h(V(t)) \nsubseteq V\left(G_{1}\right)$ 
and $h(V(t)) \nsubseteq V\left(G_{2}\right)$. Therefore $h(s)=\infty=h(t)$. If $h(V(t)) \subseteq V\left(G_{2}\right)$, then $h(V(s)) \subseteq V\left(G_{2}\right)$. Hence $h(s)=h_{2}(s)=h_{2}(t)=h(t)$.

Lemma 3.3. For a class $\mathcal{R}$ of universal graphs we have $I U_{f} P_{h} \mathcal{R} \subseteq \operatorname{Qvar}(\mathcal{R})$.

Proof. 1) Let $B \subseteq \prod_{i \in I} G_{i}$ be a homogeneous subproduct, $G_{i} \in \mathcal{R}, i \in I$ and let $q=(\Sigma \rightarrow s \approx t) \in Q i d \mathcal{R}$. We show that $B \models q$. Let $h: V(q) \rightarrow$ $V(B) \cup\{\infty\}$ be some assignment such that $B \models h(\Sigma)$. We have to show $h(s)=h(t)$. Let $h_{i}$ be a composition of $h$ and $p_{i}$ be the $i$-th projection. By Lemma 3.1, we obtain $G_{i} \models h_{i}(\Sigma)$. Thus $G_{i} \models h_{i}(s) \approx h_{i}(t)$ for all $i \in I$ because $G_{i} \models q$. Again by Lemma 3.1, we get $h(s)=h(t)$. Consequently, $B \models q$.

2) Let $G=G_{1} \cup G_{2}$ be the disjoint union of $G_{1}, G_{2} \in \mathcal{R}$ and let $q=(\Sigma \rightarrow$ $s \approx t) \in Q i d \mathcal{R}$. We show that $G \models q$. Let $h: V(q) \rightarrow V(G) \cup\{\infty\}$ be an assignment and assume $G \models h(\Sigma)$. We have to show $G \models h(s) \approx h(t)$. Define the assignments $h_{1}: V(q) \rightarrow V\left(G_{1}\right) \cup\{\infty\}, h_{2}: V(q) \rightarrow V\left(G_{2}\right) \cup\{\infty\}$ by setting $h_{i}(x)=h(x)$ if $x \in V\left(G_{i}\right)$ and $h(x)=\infty$ otherwise $(i \in\{1,2\})$. By Lemma 3.2, we get $G \models h(\Sigma)$ and $G_{i} \models h_{i}(\Sigma)$ for each $i$. Since $q \in$ QidR and $G_{1}, G_{2} \in \mathcal{R}$ (i.e. $\left.G_{i}=q\right), G_{i}=h_{i}(s) \approx h_{i}(t)$. And again by Lemma 3.2 , $G \models h(s) \approx h(t)$. Hence $G \models q$.

3) By 1) and 2), we have $I U_{f} P_{h} \mathcal{R} \subseteq Q \operatorname{var}(\mathcal{R})$.

Now we are already to formulate the main theorem for quasi-varieties in $\mathcal{G}_{u f}$.

Theorem 3.1. Let $\mathcal{R}$ be a non-empty subclass of $\mathcal{G}_{\text {uf }}$. Then $\operatorname{Qvar}_{\mathcal{G}_{u f}}(\mathcal{R})=$ $I U_{f} P_{h f} \mathcal{R}$.

Proof. Because of Lemma 3.3 and Proposition 3.1, it suffices to show $\operatorname{Qvar}_{\mathcal{G}_{u f}}(\mathcal{R}) \subseteq$ $I U_{f} P_{h} \mathcal{R}$. Since every universal graph is the disjoint union of its connected components, it remains to show $G \in I P_{h} \mathcal{R}$ for every given connected undirected universal graph $G \in \operatorname{Qvar}_{\mathcal{G}_{u f}}(\mathcal{R})$. Let $V(G)=\left\{a_{0}, \ldots, a_{n}\right\}$. Consider the following set $\Sigma$ of identities

$$
\begin{aligned}
\Sigma= & \left\{\left(x_{j_{1}}, \ldots, x_{j_{n_{i}}}\right) \approx x_{j_{1}} \mid\left(a_{j_{1}}, \ldots, a_{j_{n_{i}}}\right) \in E(G)\right\} \\
& \cup\left\{\left(x_{j_{1}}, \ldots, x_{j_{n_{i}}}\right) \approx \infty \mid\left(a_{j_{1}}, \ldots, a_{j_{n_{i}}}\right) \notin E(G)\right\} .
\end{aligned}
$$

Then $\Sigma$ is finite since $G$ is finite. Obviously, under the canonical assignment $g$ : $x_{i} \mapsto a_{i}(i=0, \ldots, n)$ we have $G \models g(\Sigma)$. Thus for given $a_{i}, a_{j} \in V(G), a_{i} \neq a_{j}$, the quasi-identity $q=\Sigma \rightarrow x_{i} \approx x_{j}$ does not hold in $G$. Since $G \in \operatorname{Qvar}(\mathcal{R})$, we have $q \notin \operatorname{Qid}(\mathcal{R})$, i.e. $\mathcal{R} \not \models q$. Hence there is some $R \in \mathcal{R}$ with $R \not \models q$, i.e. there must be some assignment $h: V(\Sigma) \rightarrow V(R) \cup\{\infty\}$ such that $R \models h(\Sigma)$ but $R \not \models h\left(x_{i}\right) \approx h\left(x_{j}\right)$. By construction of $\Sigma$ and connectedness 
of $G$, for every two variables $x, y \in V(\Sigma)$ there is some sequence $\left(z_{1}, \ldots, x\right) \approx$ $z_{1}, \ldots,\left(z_{m}, \ldots, z_{m-1}\right) \approx z_{m},\left(y, \ldots, z_{m}\right) \approx y$ of identities from $\Sigma$. Consequently, $h(x)=\infty$ would implies $h(y)=\infty$ (note $z \infty=\infty)$. Since $h\left(x_{i}\right) \neq h\left(x_{j}\right)$ we have $h(x) \neq \infty$ for all $x \in V(\Sigma)$, i.e. $h$ maps $V(\Sigma)$ into $V(R)$. By construction of $\Sigma, h^{\prime}=g^{-1} h: a_{i} \mapsto h\left(x_{i}\right)$ is a strong homomorphism from $G$ into $R$; in fact, $\left(a_{k}, \ldots, a_{l}\right) \in E(G)$ implies $\left(x_{k}, \ldots, x_{l}\right) \approx x_{k} \in \Sigma$ implies $R=\left(h\left(x_{k}\right), \ldots, h\left(x_{l}\right)\right) \approx h\left(x_{k}\right)$, i.e. $\left(h^{\prime}\left(a_{k}\right), \ldots, h^{\prime}\left(a_{l}\right)\right) \in E(R)$. Analogously, we can show that $\left(a_{k}, \ldots, a_{l}\right) \notin E(G)$ implies $\left(h^{\prime}\left(a_{k}\right), \ldots, h^{\prime}\left(a_{l}\right)\right) \notin E(R): k, l \in$ $\{0, \ldots, n\}$. By Proposition 3.1, we can conclude $G \in I P_{h} \mathcal{R}$.

Let us consider what happens if we want to treat also infinite universal graphs. Since every finitely generated subgraphs of a universal graph algebra is finite, we have $\operatorname{Qvar}(\mathcal{R})=\operatorname{Qvar}\left(S_{f}(\mathcal{R})\right)$ for a given class $\mathcal{R}$ of universal graphs, where $S_{f} \mathcal{R}$ denotes the class of all finite induced subgraphs of members of $\mathcal{R}$. Thus, for an arbitrary $\mathcal{R} \subseteq \mathcal{G}_{u}$, we have $\operatorname{Qvar}_{\mathcal{G}_{u f}}(\mathcal{R})=I S_{f} U_{f} P_{h f}(\mathcal{R})=$ $I U_{f} P_{h f} S_{f}(\mathcal{R})$ by Theorem 3.1. Moreover, we have $G \in Q \operatorname{var}(\mathcal{R})$ if and only if $S_{f}(\{G\}) \subseteq Q \operatorname{var}_{\mathcal{G}_{u f}}$. This characterizes general universal graph quasi-varieties and our restriction to finite universal graphs was not essential.

\section{Examples}

Definition 4.1. For a set $\Sigma$ of identities and classes $\mathcal{R}$ and $\mathcal{G}$ of universal graphs let $\operatorname{Mod}(\Sigma)=\left\{G \in \mathcal{G}_{d} \mid G \models \Sigma\right\}, \operatorname{Id}(\mathcal{R})=\{s \approx t \mid \mathcal{R} \models s \approx t, s, t \in$ $\left.W_{\tau}(X)\right\}, \operatorname{Var}(\mathcal{R})=\operatorname{Mod}(\operatorname{Id}(\mathcal{R})$ ) (the universal graph variety generated by $\mathcal{R}) \operatorname{Var}_{\mathcal{G}}(\mathcal{R})=\mathcal{G} \cap \operatorname{Var}(\mathcal{R})$ (the universal graph variety generated by $\mathcal{R}$ in $\mathcal{G}$ ).

Many universal graph theoretic properties can be expressed as identities or quasi-identities. We mention here some examples which may be of universal graph theoretic interest, as well.

Example 4.1. Let $\Sigma=\left\{\left(x, x_{1}, \ldots, x_{n_{i}-2}, y\right) \approx \infty, i \in I\right\}$. Then $\operatorname{Mod}(\Sigma)$ is the indicated class of universal graphs without loops.

Example 4.2. Let $\Sigma=\left\{\left(x, x_{1}, \ldots, x_{n_{i}-2},\left(y, x_{n_{i}-2}, \ldots, x_{1}, x\right)\right) \approx\left(x, x_{1}, \ldots, x_{n_{i}-2}\right.\right.$ $, y) \approx \infty, i \in I\}$. Then $\operatorname{Mod}(\Sigma)$ is the indicated class of undirected universal graphs.

Every universal graph variety is also a universal graph quasi-variety. The converse is not true, and we mention here some universal graph quasi-variety of undirected universal graphs without loops which are not universal graph varieties. 
Example 4.3. Let $C \subseteq \mathcal{G}_{u f}$ be a connected universal graph without loops. Up to isomorphism we can assume $V(C) \subseteq\left\{x_{0}, x_{1}, \ldots\right\}$ and $\left(x_{0}, x_{j_{1}}, \ldots, x_{j_{n_{i}-2}}\right.$, $\left.x_{1}\right) \in E(C), i \in I$. Let $q=\left\{\left(x_{0}, x_{j_{1}}, \ldots, x_{j_{n_{i}-2}}, x_{0}\right) \approx \infty,\left(x_{0}, x_{k_{1}}, \ldots, x_{k_{n_{i}-2}},\left(x_{1}\right.\right.\right.$ $\left.\left.\left., x_{k_{n_{i}-2}}, \ldots, x_{k_{1}}, x_{0}\right)\right) \approx\left(x_{0}, x_{k_{1}}, \ldots, x_{k_{n_{i}-2}}, x_{1}\right), \Sigma(C) \rightarrow x_{0} \approx x_{1}, i \in I\right\}$, where $\left.\Sigma(C)=\left(x, x_{j_{1}}, \ldots, x_{j_{n_{i}-2}}, y\right) \approx x \mid\left(x, x_{j_{1}}, \ldots, x_{j_{n_{i}-2}}, y\right) \in E(C)\right\} \cup\left\{\left(x, x_{k_{1}}, \ldots\right.\right.$ $\left.\left.x_{k_{n_{i}-2}}, y\right) \approx \infty \mid\left(x, x_{k_{1}}, \ldots, x_{k_{n_{i}-2}}, y\right) \notin E(C)\right\}, x, y \in V(C), i \in I$. We see that $\operatorname{Mod}(q)$ is the class of undirected universal graphs without loops which contain no induced subgraph isomorphic to $C$ or to a strong homomorphism image of $C$. So $\operatorname{Mod}(q)$ is a universal graph quasi-variety but it is no a universal graph variety.

\section{Conclusion and discussion}

In this article, a universal graph theoretic characterization of universal graph quasi-varieties, i.e. the classes of universal graphs with can be defined by quasi-identities for their corresponding universal graph algebras, is given. We obtain a theorem of Birkhoff-type, which uses graph theoretic closure operations. A class of finite undirected universal graphs is quasi-equational (i.e., a universal graph quasi-variety) if and only if it is closed with respect to isomorphisms, induced subgraphs, finite disjoint unions and homogeneous subproducts. Finally, it is worth while to mention that the result can be a guideline for futher investigate in the directions of the equational logic for universal graph algebras, universal graph varieties, subvarieties, and subvarieties of universal graph varieties generated by universal graph algebras.

\section{Acknowledgment}

This work was supported by the Higher Education Research Promotion and National Research University Project of Thailand, Office of the Higher Education Commission, through the the Cluster of Research to Enhance the Quality of Basic Education.

\section{References}

[1] S. Burris and H.P. Sankappanavar, A course in Universal Algebra, Springer-Verlag New York Heidelberg Berlin 1981.

[2] K. Denecke and S.L. Wismath, Universal Algebra and Applications in Theoretical Computer Science, ChapmanHall/CRC 2002.

[3] T. Poomsa-ard, Identities in universal graph algebras, Int. Math. Forum, 4, 2009, no. 35, 1715-1722. 
[4] R. Pöschel and W. Wessel, Classes of graphs definable by graph algebras identities or quasiidentities, Comment. Math. Univ. Carolonae 28(1987), 581-592.

[5] C. R. Shallon, Nonfinitely based finite algebras derived from lattices, $\mathrm{Ph}$. D. Dissertation, Uni. of California, Los Angeles, 1979.

Received: February 21, 2013 